\title{
Malignant ventricular tachycardia secondary to topiramate induced refractory hypokalemia
}

\begin{abstract}
Topiramate is a well-known, effective neuromodulator used in the treatment of seizure disorder and prophylaxis of migraines. There have been a small number of case reports and studies in the literature that demonstrate the effects of topiramate on acid- base balance and hypokalemia, but none has reported on the potential deadly consequences of using this medication in patients at risk for malignant arrhythmias. We present a case of a Caucasian female with Non-ischemic cardiomyopathy on topiramate therapy for migraine prophylaxis that developed life-threatening ventricular tachycardia storm leading to acute decompensated heart failure in the setting of topiramate-induced refractory hypokalemia. In this case report, we propose the avoidance of topiramate and other neuromodulators that predispose patients with migraines and seizure disorder to hypokalemia who also have known risk factors for malignant arrhythmias.
\end{abstract}

Keywords: topiramate, malignant ventricular arrhythmias, non-ischemic cardiomyopathy
Volume 8 Issue 4 - 2018

Roland Njei, Hannah Hope Pharm D

Department of Internal Medicine, Washington University St Louis/Barnes Jewish Hospital, USA

Correspondence: Roland Njei, Department of Internal Medicine, Washington University St Louis/Barnes Jewish Hospital, USA, Email rnjei@wustl.edu

Received: July 26, 2018 | Published: August 02, 2018
Abbreviations: VT, ventricular tachycardia; CRT-D, cardiac resynchronization therapy; $\mathrm{CCU}$, cardiac intensive care unit; $\mathrm{K}$, Potassium; CA, carbonic anhydrase; TPM, topiramate; BMP, basic metabolic panel.

\section{Introduction}

Topiramate (TPM) is a well-known and effective neuromodulator used in the treatment of seizure disorder and prophylaxis of migraines. The precise mechanisms of its therapeutic benefits are unknown. However, it is hypothesized through preclinical studies that it exerts its effects through multiple mechanisms of action that include voltage-dependent sodium channel blockade, glutamate inhibition, $\gamma$-aminobutyrate potentiation, and inhibition of the carbonic anhydrase enzyme. In controlled trials, topiramate has been associated with changes in several clinical chemistry laboratory measurements, including a decrease in serum potassium $(0.4 \%$ topiramate versus $0.1 \%$ placebo). We present a case of a Caucasian female with non-ischemic cardiomyopathy on topiramate therapy for migraine prophylaxis who developed life-threatening sustained ventricular tachycardia (VT) in the setting of refractory hypokalemia. In this case report, we propose the avoidance of topiramate and other neuromodulators that predispose patients with migraines and seizure disorder who also have known risk factors for malignant arrhythmias.

\section{Clinical summary}

A 52 year old Caucasian female with a history of non-ischemic cardiomyopathy secondary of valvular disease (EF 26\%) status post Cardiac Resynchronization Therapy Device (CRT-D), status post tricuspid annuloplasty with severe tricuspid regurgitation, mitral valve replacement with a St Jude's valve in 2010, atrial fibrillation status post a MAZE procedure in 2010, obstructive sleep apnea (OSA) on CPAP, hypothyroidism, HTN, migraines, tobacco abuse who presented to an outside hospital with palpitation, shortness of breath, chest pain and more than 10 ICD shocks. She was found to be in a VT storm; initial EKG showed sustained polymorphic VT but no concern for an acute coronary ischemic event. Labs were significant for a serum
$\mathrm{K}$ of 2.2. She was given $80 \mathrm{mg}$ IV K and $4 \mathrm{gm}$ of Magnesium and air lifted to the cardiac intensity care unit at our medical center (CCU). On arrival to our CCU, she was found to again be in VT and was shocked by her ICD. She briefly became unresponsive and required chest compressions, was shocked again by her ICD and regained consciousness. She received an amiodarone bolus followed by an amiodarone drip (gtt). She continued to have VTs requiring shocks from her ICD. She was then given a bolus of lidocaine and also started on a lidocaine drip.

Initial CCU labs on arrival showed a serum $\mathrm{K}$ of 2.3, Bicarbonate of 33, serum Cr of 1.2 (baseline $~ 1.1$ ), serum magnesium of 2.6, $\mathrm{PH}$ of 7.29 and a proBNP 3192 . She continued to have multiple episodes of sustained VT requiring shocks from her ICD as well as external defibrillation and then progressed to a hemodynamically unstable state. She was intubated and required Levophed for pressure support once sedation was initiated. A central line was placed, and her serum $\mathrm{K}$ was aggressively repleted requiring over $200 \mathrm{mEq} / \mathrm{L}$ of potassium over 24 hours. On review of her home medications, she was found to be on torsemide $40 \mathrm{mg}$ daily, metolazone $2.5 \mathrm{mg}$ daily, and topiramate $100 \mathrm{mg}$ extended release daily that was started one month prior to presentation. With normalization of her serum potassium, she had no more episodes of VT and she was extubated the next day. She was found to have a low forward flow state and volume overload. She was then aggressively diuresed with $80 \mathrm{mg}$ of IV furosemide and monitored with serial basic metabolic panel (BMP) every 6-8hours. She responded well to IV diuresis with improvement in her hemodynamic states and was weaned of pressors. With her serum $\mathrm{K}$ within normal limits, she had no more episodes of VT and was transitioned from her amiodarone and lidocaine drip to oral amiodarone 400mg TID. Throughout her CCU course, she was kept on her topiramate as there was initially less concern that it was the driving force behind the refractory hypokalemia. With improvement in clinical status patient was moved from the CCU to the floor for a few more days of diuresis prior to discharge home. While on the floor, her serum $\mathrm{K}$ was now monitored and repleted with daily basic metabolic panel (BMP) and unfortunately 2days after being on the floor, she had 3 more episodes 
of sustained VT requiring 3 ICD shocks accompanied by temporary unresponsiveness as well as hemodynamic instability. A point of care serum potassium was checked, and it was 2.2 despite being above 4 at the time of transfer out of the CCU.

She was again transferred back to the CCU for further management In the CCU, her potassium was again aggressively repleted with both oral and IV K; she was completely taken off topiramate. With normalization of her serum $\mathrm{K}$, she was later transferred back to the floor after a one day in the CCU. She was observed on the floor for two more days and discharged home without any more VT episodes. At time of discharge, her serum potassium was 4.1. Plan was for her to follow up with her neurologist outpatient to be started on another anti-migraine neuromodulator without a similar side effect profile to topiramate. Outpatient follow up off Topiramate showed continuously stable serum potassium as shown in the graphs below (Figure 1) (Figure 2).

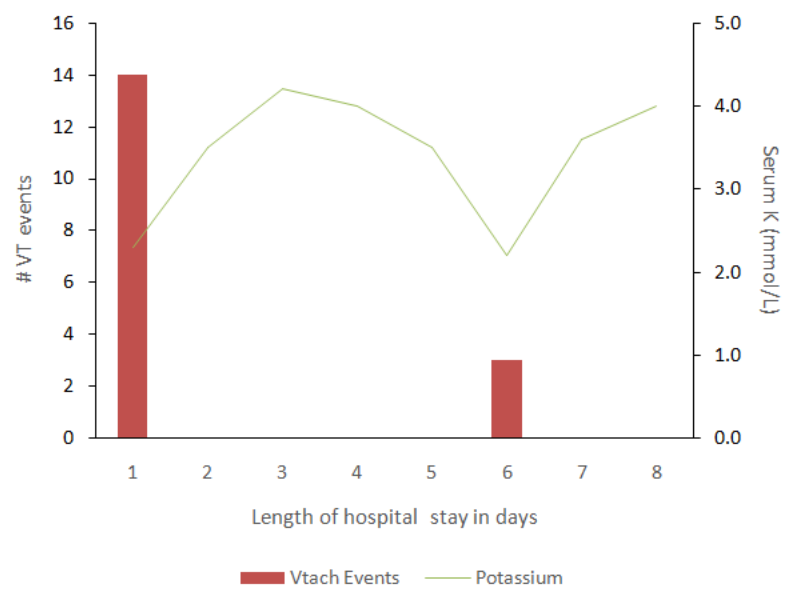

Figure I Serum K+\&VT episodes requiring shocks while on Topiramate.

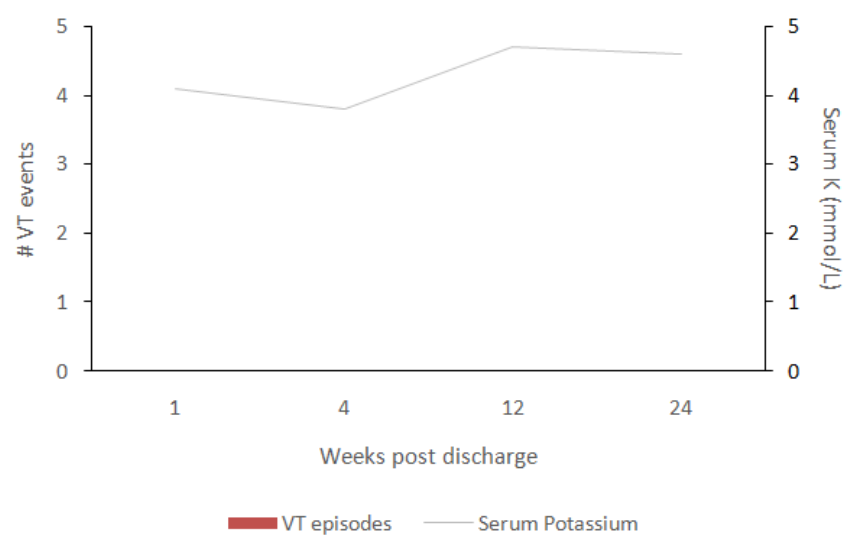

Figure 2 Serum $\mathrm{K}+\& \mathrm{VT}$ episodes requiring shocks off Topiramate postdischarge.

\section{Discussion}

Topiramate is a sulfamate-substituted monosaccharide that is currently used as monotherapy or adjunctive therapy for seizure disorder and migraine prophylaxis. It is structurally different from other antiepileptic drugs and has several mechanisms of action, including inhibition of voltage-dependent sodium and L-type voltage- gated calcium channels, enhancement of GABA-mediated inhibition, decrease glutamate-mediated excitatory neurotransmission, and inhibition of carbonic anhydrase (CA) isoenzymes especially in the proximal convoluted tubules of the kidney. ${ }^{1-10}$ Carbonic anhydrase (CA) is responsible for bicarbonate reabsorption by proximal tubule cells and urinary acidification by distal tubules. Inhibition of carbonic anhydrase can cause a metabolic acidosis by impairing normal reabsorption of filtered bicarbonate by proximal tubule and excretion of $\mathrm{H}+$ by the distal tubule. Bicarbonate loss into the urine increases the negativity of the renal tubule lumen resulting in increased potassium secretion into the lumen which then leads to hypokalemia and mixed renal tubular acidosis (RTA). Hypokalemia is an independent risk factor contributing to reduced survival of cardiac patients and increased incidence of fatal arrhythmias. The destabilization of potassium homeostasis may have negligible effect in some patients but there is a significant number of individuals with underlying cardiomyopathy who are at risk of malignant arrhythmias as in our patient.

Potassium homeostasis play a critical part in normal cellular function and is under the control of ion-exchange pumps (cellular, membrane-bound, sodium-potassium ATPase pumps). Approximately $90 \%$ of total body potassium is intracellular while $10 \%$ is extracellular of which less than $1 \%$ is composed of plasma. The ratio of intracellular to extracellular potassium governs neuromuscular and cardiovascular excitability. Potassium homeostasis is regulated by two systems; the first regulates $\mathrm{K}+$ absorption and excretion via the kidneys and intestines (external balance). The second regulates $\mathrm{K}+$ shifts between the intracellular and extracellular space (internal balance). Internal balance is under the control of insulin and catecholamines. Both the internal and external balance are affected by many medications such as Topiramate as in our case but the degree of influence and resulting outcome is variable. ${ }^{5,8}$ Potassium derangements often lead to neuromuscular, gastrointestinal and most importantly cardiac rhythm abnormalities. Several case reports and clinical trials have validated metabolic acidosis and hypokalemia as possible side effects of topiramate, but the effect of this potassium disturbance has always been reported as mild. However, given the possibility of polymorphisms in the carbonic anhydrase gene such as subtype 12, it is important to note that the degree and consequences of the hypokalemia are going to vary. ${ }^{89}$ This may range from asymptomatic hypokalemia as observed in prior case reports to malignant arrhythmia as seen in our patient.

Hypokalemia-induced arrhythmogenicity occurs via the following three mechanisms; slowed conduction, pacemaker abnormality and prolongation of the ventricular repolarization. Slowed conduction is due to membrane hyperpolarization which results in increased excitatory threshold. Prolongation of ventricular repolarization in the setting of hypokalemia is caused by inhibition of outward potassium current associated with increased propensity for early after depolarizations. Pacemaker dysfunction is caused by increasing the slope of diastolic depolarization in the Purkinje fibers, as well as delayed after depolarizations caused by calcium overload secondary to sodium/ potassium pump blockade and sodium/calcium exchange reverse mode stimulation. ${ }^{9,11,12}$ The effect of hypokalemia on repolarization is not uniform at every ventricular sites leading to amplified spatial repolarization gradients which promote unidirectional conduction block. With hypokalemia, the prolongation of action potential may be associated with shortening of the effective refractory period, thus increasing the propensity for ventricular re-excitation over late phase of repolarization. Early and delayed after depolarizations, oscillatory 
prepotentials in Purkinje fibers and a favorable electrophysiological substrate unidirectional conduction block, reduced excitation wavelength, increased interval for ventricular re-excitation may account for the mechanism of life-threatening tachyarrhythmias in hypokalemic patients. ${ }^{6,12}$

Prevention and treatment going forward should include thorough review of home medications and medical history. Avoidance of neuromodulators with a propensity for destabilization of potassium as well as acid-base homeostasis should be the standard of care in patients with known ischemic and non-ischemic cardiomyopathy as there is evidence of possible polymorphisms in the carbonic anhydrase gene such as subtype 12 which increase the risk of hypokalemia induced malignant arrhythmias. Patients with cardiovascular risk factors for fatal arrhythmias found to be on a neuromodulator such as topiramate that predisposes to potassium or other electrolyte disturbances should be switched to other medications with similar neurological effects but little or no effect on potassium or acid-base homeostasis.

\section{Acknowledgments}

None.

\section{Conflict of interest}

Author declares no conflict of interests.

\section{References}

1. Nadkarni GN, Annapureddy N, Meisels IS. Topiramate-Induced Refractory Hypokalemia: a case report. Am J ther. 2014; 21(5):e157-e158.

2. Eva A. Belotti, Ilaria Taddeo, et al. Chronic impact of topiramate on acid-base balance and potassium in childhood. Eur J Paediatr Neurol. 2010;14(5):445-448.
3. Mirza N, Marson AG, Pirmohamed M. Effect of topiramate on acidbase balance: extent, mechanism and effects. $\mathrm{Br} J$ Clin Pharmacol. 2009;68(5):655-661.

4. Laing CM, Unwin RJ. Renal tubular acidosis. J Nephrol. 2006;19(supp1 9):S46-S52.

5. Shank RP, Gardocki JF, Streeter AJ, and Maryanoff, B.E. An overview of the preclinical aspects of topiramate (pharmacology, pharmacokinetics, and mechanism of action). Epilepsia. 2000;41:S3-S9.

6. Stowe CD, Bollinger T, James LP, et al. Acute mental status changes and hyperchloremic metabolic acidosis with long-term topiramate therapy. Pharmacotherapy. 2000;20(1):105-109.

7. Dell'Orto VG, Belotti EA, Goeggel-Simonetti B, et al. Metabolic disturbances and renal stone promotion on treatment with topiramate: a systematic review. Br J Clin Pharmacol. 2014;77(6):958-964.

8. Shiber JR. Severe non-anion gap metabolic acidosis induced by topiramate: a case report. J Emerg Med. 2010;38:494-496.

9. Jovanović M1, Sokić D, Grabnar I, et al. Effect of Long-term Topiramate Therapy on Serum Bicarbonate and Potassium Levels in Adult Epileptic Patients. Ann Pharmacother. 2014;48(8):992-997.

10. Girish N, Nadkarni, Narender Annapureddy, et al. TopiramateInduced Refractory Hypokalemia. American Journal of Therapeutics. 2014;21(5):e157-e158.

11. Nguyen FN, Kar JK, Verduzco-Gutierrez M, et al. A Case of Hypokalemic Paralysis in a Patient with Neurogenic Diabetes Insipidus. The Neurohospitalist. 2014;4(2):90-93.

12. Oleg E. Osadchii. Mechanisms of hypokalemia-induced ventricular arrhythmogenicity. Fundamental \& clincal pharmacology. 2010;24(5):547-559. 